\title{
FIREcast system - previsional fire danger index computation system for alpine regions
}

\author{
L. Corgnati, M. Gabella \& G. Perona \\ Dipartimento di Elettronica, Politecnico di Torino, Torino, Italy
}

\begin{abstract}
FIREcast is a previsional fire danger index computing system, which elaborates weather parameter maps to evaluate the danger indicator on the interest area. FIREcast uses as a starting point the foreseen Canadian Fire Weather Index (FWI) adjusted for continental European latitudes and climatology and adapted for alpine regions orography. As FWI is a meteorological fire danger index, it represents the fire danger level due solely to the present and past weather conditions, not considering contingent human presence and actions. FIREcast operates on meteorological forecast input data maps, to obtain output maps representing expected fire danger in the studied area (Piedmont criteria in the current case) with a forecasting time interval up to 72 hours, after adjusting the forecast to historical fire records. FIREcast is a valuable aid in firefighting management: it allows involved agencies to have an efficient resources administration (both logistic and human), well aimed territory monitoring and reliable intervention planning for operators' safety.
\end{abstract}

Keywords: forest fires, forecast, fire danger index, alpine regions.

\section{Introduction}

The first crucial step in forest fires management is the identification of those parameters that allow for the estimation of fire rising probability. Canadian Fire Weather Index (FWI) is a powerful instrument to analyse such a problem, with reliable performances also at our latitudes. FWI computes six indices to characterize fire rising probability and turn-on parameters. Inputs are daily air temperature, air relative humidity, wind speed and rainfall measurements from a ground station network. To compute the final indices, the also method uses the historical evolution of these quantities. The FWI method has been developed 
specifically for the Canadian environment, namely for its mostly flat orography and for its climate properties bearing to an April-to-November fire season. Consequently, FWI is not suited in its original form to the European environment (particularly alpine regions); indeed the April-to-November period matches the Mediterranean regions fire season, while continental and alpine European zones present a fall-winter fire season. Hence it is necessary to review the method itself, in order to suit the FWI indices to European continental weather, climatology, vegetation and orography. Canadian plane orography makes single ground station data reliable for a large homogeneous area; alpine regions' complex orography involves a different and more sophisticated model input data evaluation. It would be useful to obtain the algorithm inputs by the application of forecasting models on the studied regions. In such a way the method could operate on mesoscale, easy-to-pick and reliable input data with the possibility of working in a forecasting direction. This work describes the implementation of software able to compute a global forecast fire danger index based on the FWI method adapted for the European alpine region environment (with a forecasting time interval of up to 72 hours). The application has been developed within a Regione Piemonte project aiming at the realization of a Piedmont-adapted fire weather index computing system. The following sections describe FIREcast: Section 2 explains the theoretical concepts, Section 3 describes data structure, Section 4 examines algorithm structure, Section 5 illustrates the validation procedure and results.

\section{The concept}

As explained in [1], FWI uses six standard parameters. The first three are fuel moisture codes that follow daily changes in the moisture content of three classes of forest fuels with different drying rates. The last three components are fire behaviour indices representing the rate of spread, assumed fuel weight consumed and fire intensity. The moisture parameters are arranged in code form, with values rising as moisture content decreases. The three moisture codes and their corresponding fuels are:

- Fine Fuel Moisture Code (FFMC), which represents the moisture content of litter and other cured fine fuels in a forest stand in a layer of dry weight about $0.25 \mathrm{~kg} / \mathrm{m}^{2}$.

- Duff Moisture Code (DMC), which represents the moisture content of loosely compacted decomposing organic matter weighing about 5 $\mathrm{kg} / \mathrm{m}^{2}$ when dry.

- Drought Code (DC), which represents a deep layer of compact organic matter weighting about $25 \mathrm{~kg} / \mathrm{m}^{2}$ when dry.

The three moisture codes plus wind are linked in pairs to form two intermediate indices that are in turn combined to yield the final index, the FWI. These last three components are:

- Initial Spread Index (ISI), a combination of wind and FFMC, which represents the rate of spread alone, without the influence of variable quantities of fuel. 
- Build Up Index (BUI), a combination of DMC and DC, which represents the total fuel available to the spreading fire.

- Fire Weather Index (FWI), a combination of ISI and BUI, which represents the intensity of the spreading fire as an energy output rate per unit length of fire front (Byram's equation).

The index evaluation depends solely on weather readings taken each day at noon local standard time (LST), with the following accuracy (related to measuring instruments):

- $\quad \mathbf{T}\left[{ }^{\circ} \mathrm{C}\right]$ : air temperature at noon; required accuracy: $\pm \mathbf{0 . 5} \mathbf{5}^{\circ} \mathbf{C}$.

- $\quad \mathbf{H}[\%]$ : relative humidity at noon; required accuracy: $\pm \mathbf{1 \%}$

- $\mathbf{W}[\mathrm{km} / \mathrm{h}]$ : wind speed at noon; required accuracy: $\pm \mathbf{1} \mathbf{~ k m} / \mathbf{h}$.

- $\quad \mathbf{r}_{\mathbf{0}}[\mathrm{mm}]$ : daily rainfall amount; required accuracy: $\pm \mathbf{0 . 2} \mathbf{~ \mathbf { m }} / \mathbf{h}$.

As said in the abstract, the FWI index has been implemented for the specific Canadian environment: different danger level behaviour stems from environmental differences between Canadian territory and European alpine regions: namely different orographic conditions and, above all, different latitudes. Northernmost Canadian latitudes bear different irradiation conditions, which involve different climatology and all consequent mismatches in atmospheric parameters used as input in the FWI system. Main differences have a radiative nature, due to the different latitudes and are thus affect the atmospheric parameters involved in fuel moisture content evaluation. Minor divergences are detectable in precipitation amounts and wind speed. Table 1 summarises the environmental gap between Canadian and continental European atmospheric parameters (Canadian latitudes considered [48-65] deg, continental Europe latitudes considered [42-50] deg).

Table 1: $\quad$ Main atmospheric parameter differences due to the environmental gap between Canada and continental Europe. Differences have been intended as continental European values minus Canadian values.

\begin{tabular}{|l|l|}
\hline Quantity & Difference range \\
\hline SOLAR RADIATION & $50 \div 150 \mathrm{~W} / \mathrm{m}^{2}$ \\
\hline NET THERMAL FLUX & $20 \div 40 \mathrm{~W} / \mathrm{m}^{2}$ \\
\hline $\begin{array}{l}\text { COLUMN INTEGRATED WATER } \\
\text { VAPOUR }\end{array}$ & $5 \div 10 \mathrm{~kg} / \mathrm{m}^{2}$ \\
\hline SURFACE TEMPERATURE & $5 \div 20^{\circ} \mathrm{C}$ \\
\hline EVAPORATION minus PRECIPITATION & $0.2 \div 4 \mathrm{~mm} /$ day \\
\hline PRECIPITATION & $\approx 0 \mathrm{~mm} /$ day \\
\hline WIND SPEED & $\approx-2 \mathrm{~m} / \mathrm{s}$ \\
\hline
\end{tabular}

Primarily, FFMC and then DMC and DC are the most influenced parameters, because they describe different types of fuel moisture and are strictly dependent on radiative factors, especially on atmospheric water vapour content and temperature. Hence computing algorithm primary adjustments need to be aimed at the calibration of fuel moisture codes (FFMC, DMC, DC). Other partial 
indices (ISI and BUI) do not depend directly on moisture content or temperature, but stem from fuel codes and wind, the only parameter not to be influenced by climate differences.

\subsection{Fine fuel moisture modelling}

As explained in $[7,8]$, the dominant physical processes controlling changes in the moisture content of fine, dead forest litter are latent heat effects, vapour exchange and precipitation Hence, implemented adjustments in fuel moisture codes concern moisture content calibration, as a function of relative humidity and temperature contributions, according to the application of correction factors expressing the mean radiative differences between the two environments. The correction terms depend on the season, time of day, slope atmospheric haze and shading by clouds and crown, as well as on air temperature, air relative humidity and wind speed. All adjustments are empirically determined. As shown in [2-5], the FFMC index uses the EMC functions of Van Wagner for desorption and absorption (eqns. (1)-(5)) for mid-afternoon fine fuel moisture content in the absence of rainfall and in a mid latitudes region.

$$
\begin{gathered}
k_{0}=0.424 \cdot\left[1-\left(\frac{H}{100}\right)^{1.7}\right]+0.0694 \cdot W^{0.5} \cdot\left[1-\left(\frac{H}{100}\right)^{8}\right] . \\
k_{d}=k_{0} \cdot 0.581 \cdot e^{0.036 \cdot T} . \\
m=E_{d}+\left(m_{0}-E_{d}\right) \cdot 10^{k_{d}} \\
m=E_{w}-\left(E_{w}-m_{0}\right) \cdot 10^{k_{d}} \quad \text { if } \mathrm{m}_{\mathrm{o}}>\mathrm{E}_{\mathrm{d} \cdot} . \\
m=m_{o} \quad \text { if } \mathrm{m}_{\mathrm{o}}<\mathrm{E}_{\mathrm{d}} \text { and } \mathrm{m}_{\mathrm{o}}<\mathrm{E}_{\mathrm{w}} \text { and } \mathrm{m}_{\mathrm{o}}>\mathrm{E}_{\mathrm{w}} .
\end{gathered}
$$

being $\mathrm{m}_{\mathrm{o}}=$ water content in fine fuel of the previous day, $\mathrm{m}=$ water content in fine fuel after the drainage, $\mathrm{k}_{\mathrm{o}}\left[\log _{10}(\mathrm{~m}) /\right.$ day $]=\log$ drying rate at the standard temperature of $21.1{ }^{\circ} \mathrm{C}, \mathrm{k}_{\mathrm{d}}\left[\log _{10}(\mathrm{~m}) /\right.$ day $]=\log$ drying rate, $\mathrm{E}_{\mathrm{w}}=$ equilibrium water content of fine fuel after moistening, $\mathrm{E}_{\mathrm{d}}=$ equilibrium water content of fine fuel after the drainage. Due to experimental studies on a chosen training dataset and according to methods depicted in $[6,9,10]$, the absorption and desorption equations have been reformulated as follows for application in alpine regions of continental Europe.

$$
\begin{gathered}
k_{d}=\left\{0.567 \cdot\left[1-\left(\frac{H}{100}\right)^{1.7}\right]+0.176 \cdot W^{0.5} \cdot\left[1-\left(\frac{H}{100}\right)^{8}\right]\right\} \cdot \\
e^{0.0355 T} . \\
m=E_{d}+\left(m_{0}-E_{d}\right) \cdot 10^{k_{d}} .
\end{gathered}
$$

for drainage conditions and

$$
\begin{aligned}
& k_{w}=\left\{0.567 \cdot\left[1-\left(\frac{100-H}{100}\right)^{1.7}\right]+0.176 \cdot W^{0.5}\right. \\
& \left.\cdot\left[1-\left(\frac{100-H}{100}\right)^{8}\right]\right\} \cdot e^{0.00355 T}
\end{aligned}
$$




$$
m=E_{w}-\left(E_{w}-m_{0}\right) \cdot 10^{k_{w}} .
$$

for moistening conditions. Temperature and relative humidity values have been adjusted according to detected climatologic differences between the two environments.

\subsection{Danger classes' bounds calibration}

The use of the five original Van Wagner classes in FIREcast software results in a very inhomogeneous event (fire events and non-fire events) distribution among classes. In particular, the original 'Very High Danger' class presents insignificant occurrence numbers, thus making a 5 class danger ranking useless. Danger classes' calibration strategy aims at the achievement, using an adaptative technique, of a four classes ranking with equally 'populous' classes and a reasonable partition between fire events and non-fires event within each class. The four defined danger classes by calibration strategy are 1-EXTREME, 2HIGH, 3-MODERATE, 4-LOW.

Table 2: $\quad$ FWI original danger classes (Van Wagner formulation) versus FIREcast defined danger classes.

\begin{tabular}{|c|c|c|c|}
\hline $\begin{array}{c}\text { FWI original danger } \\
\text { classes }\end{array}$ & $\begin{array}{c}\text { FWI } \\
\text { values }\end{array}$ & $\begin{array}{c}\text { FIREcast danger } \\
\text { classes }\end{array}$ & $\begin{array}{c}\text { FWI } \\
\text { values }\end{array}$ \\
\hline Very High & $>29.8$ & Extreme & $>13$ \\
High & $19.8 \div 29.8$ & High & $9 \div 13$ \\
Moderate & $13.3 \div 19.8$ & Moderate & $4 \div 9$ \\
Low & $5.6 \div 13.3$ & Low & $0 \div 4$ \\
Very Low & $0 \div 5.6$ & & \\
\hline
\end{tabular}

The class bounds refinement procedure is based on a test area in the Piedmont alpine region, whose climatologic, orographic and fire information for a 10 years period were available. Thresholds identifying the four defined danger classes have been monthly differentiated, in order to best fit small scale danger condition changes in time and to best use index potentialities. The monthly correction pattern represents fluctuations in time (due to the strong dependence on the season's weather) of danger conditions. The pattern has been used in order to adjust the FWI original danger classes, which have a 'mean' nature.

\section{Data structure}

To implement a global fire danger evaluating system, which is able to compute index values on the entire region of interest, it is necessary to let it operate with input data in the form of a matrix, which means a change in input dataset 
characterization from scalar and point values to raster images. Input data have to be maps of air temperature, relative humidity, wind speed and rainfall amount over the studied area and should obey FWI model data accuracy and time reference conditions. The standard $3-\mathrm{h}, 0.5^{\circ}$ resolution, 60 hybrid level, European Centre for Medium-Range Weather Forecasts (ECMWF) analyses have been chosen as input data. ECMWF data structure and details are given in [11].

\subsection{Accuracy}

As FIREcast operates on foreseen data, data accuracy requirements could not be satisfied due to the nature of the forecast model itself. A model generating foreseen data could not guarantee accuracy in terms of proper measurement precision: data are evaluated and not measured and, above all, the estimated value is computed by a forecast model. Thus, accuracy is no more of a significant parameter, at least not in its proper measurement meaning. This fact is not a crucial problem because of FIREcast's previsional complement role. Due to its previsional nature, FIREcast is not supposed to provide exact outputs to be used in any further evaluation process. FIREcast is intended to be an innovative aid in the prevention phase: it aims at addressing surveillance, monitoring and intervention procedures in order to avoid or minimize fire events and to manage safe and efficient extinguish operations. Thus, extreme precision in the previsional fire index is not necessary, as long as good protection from missing detections and false alarms is ensured.

\subsection{Resolution}

The system resolution requirements are strictly related to its applications: The strong bond linking FIREcast with field operations results in a solution to the hard trade-off: data shall guarantee a sufficiently high resolution to allow for precise positioning of alarm pixels, in order to control and direct operations with high accuracy and efficiency. At the same time, competent agencies have to manage with the few available resources (both human and logistic): assets optimization entails territory sectoring. Sectors have a large extent: thus data resolution shall not be too high, so as not to give redundant positioning information, which wouldn't be used and which may cause administrative problems, because of the presence, in a single sector, of different danger levels instead of just one reference value.

\section{FIREcast system}

This section describes FIREcast's structure, with particular focus on data structure, index adjustment models and system crucial parts: the input reading module, evaluating module and output generating module. 


\subsection{Input data reading module (IDRM)}

IDRM is the first FIREcast operating module. It receives as input foreseen maps from ECMWF, converts them into the correct format and selects the area to be analyzed. IDRM receives input data from an external interface module, which connects with the ECMWF Meteorological Archival and Retrieval Service, performs a search for climatologic parameters, the date and geographic area and downloads air temperature, dew point temperature, zonal and meridional wind components and convective and large scale precipitation data. Each data file contains all required parameter distributions over the selected area in 24 sequential forecasting levels, one every 3 hours, thus covering a foreseen interval of 72 hours. The IDRM first unit, the Input Parameter Evaluation Unit, firstly separates each parameter set in the incoming file for type and for forecasting time and saves the divided sets in single files. Afterwards, the unit processes the obtained files to evaluate the necessary parameters (air temperature, air relative humidity, total wind speed, total precipitation amount) for fire index computation and saves them in separate files (for each quantity set, three files with three different forecasting intervals). The Geographic Selection Unit operates on these files creating parameter map files just over a smaller area around Piedmont (longitude and latitude bounds are chosen by the user).

\subsection{Index evaluation module (IEM)}

IEM receives air temperature, air relative humidity, wind speed and precipitation maps from IDRM and computes the six indices of the Canadian FWI system (FFMC, DMC, DC, ISI, BUI, FWI). Output FWI values are grouped into four danger classes, whose bounds estimation algorithm applies the monthly correction pattern described in the previous Section. IEM performs FWI indices computation through its Indices Evaluation Unit, which applies the Canadian indices formulation on maps received from IDRM. For each day, the Indices Evaluation Unit computes the six indices, taking into account the previous days evaluated maps, in order to consider the historical evolution of atmospheric parameters and indices. Each intermediate index matrix is saved in a file to be stored in archives: in this way it will be possible to reproduce at anytime the indices calculation procedure and to retain memory of historical indicators evolution. Indices are computed for the forecasting time indicated by the user: in its default setting, FIREcast generates indices predictions at 24, 48 and 72 hours. Indices evaluation is performed applying the moisture content climatologic correction modelled in Eqns. (6)-(9). The Climatologic Correction Unit implements the correction procedure and runs it inside the evaluation process. Evaluated FWI index matrices are then elaborated by the Danger Classes Definition Unit, which defines the danger level bounds by applying the monthly correction pattern evaluated by the Correction Pattern Unit.

\subsection{Output generating module (OGM)}

The OGM processes intermediate data provided by IEM: the OGM Mapping Unit evaluates its inputs and generates fire danger maps, both FWI value maps 
and FWI danger level maps, for the selected forecasting intervals. All output map parameters can be defined by the user. In its default settings, the unit generates $24 \mathrm{hr}, 48 \mathrm{hr}$ and $72 \mathrm{hr}$ foreseen FWI danger classes maps. Figure 1 shows an example of an output map. The OGM Storage Unit stores the final data, in order to build an archive of fire danger level historical evolution.

\section{Results and validation}

Available data for validation procedures concerns historical series of fire events and weather forecasted maps for the period 1998-2005. In order to obtain validation results that are reliable and not dependent on the proper structure of the computational system, the dataset has been divided into two different sets, one for intermediate verification procedures and one for final verification procedures.

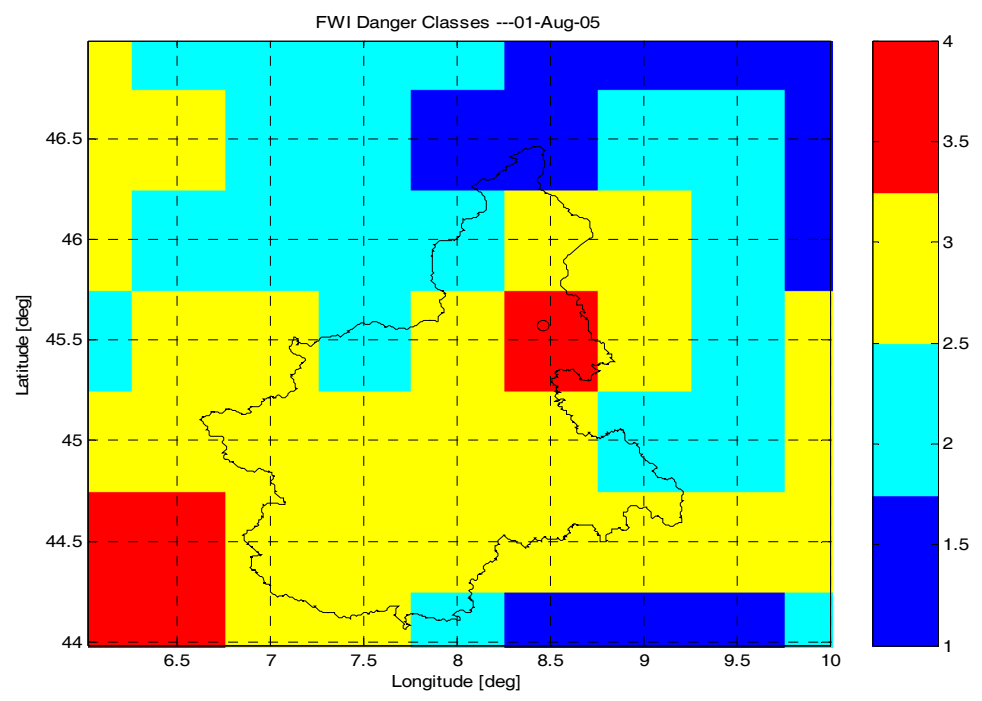

Figure 1: $\quad$ FIREcast output maps for March $7^{\text {th }}, 2005$ (24 hour forecast). Circles indicate fire event positions. Classes: 1 (black) - low, 2 (dark) - moderate, 3 (bright) - high, 4 (white) - extreme.

Intermediate validations have been performed on the Training Dataset, defined as a set of historical series of fire events and weather forecasted maps for the period 1998-2000. Final validations have been performed on the Validation Dataset, defined as a set of historical series of fire events and weather forecasted maps for period 2001-2005. Input foreseen maps are affected by spatial variability tied to the weather forecast. By considering this issue, FIREcast achieves amazingly good results, as shown in Figure 2. The spatial variability oriented validation technique is based on the comparison of each fire position 
with the mean trend of danger level in the corresponding pixel and in the 8 pixels surrounding the corresponding pixel. The huge improvement in validation results with respect to a classical severe validation, underlines that input data spatial variability is a crucial issue: often the 'correct' danger level is related to a pixel adjacent to the corresponding pixel. By correcting this offset, FIREcast becomes a very powerful forecasting instrument.
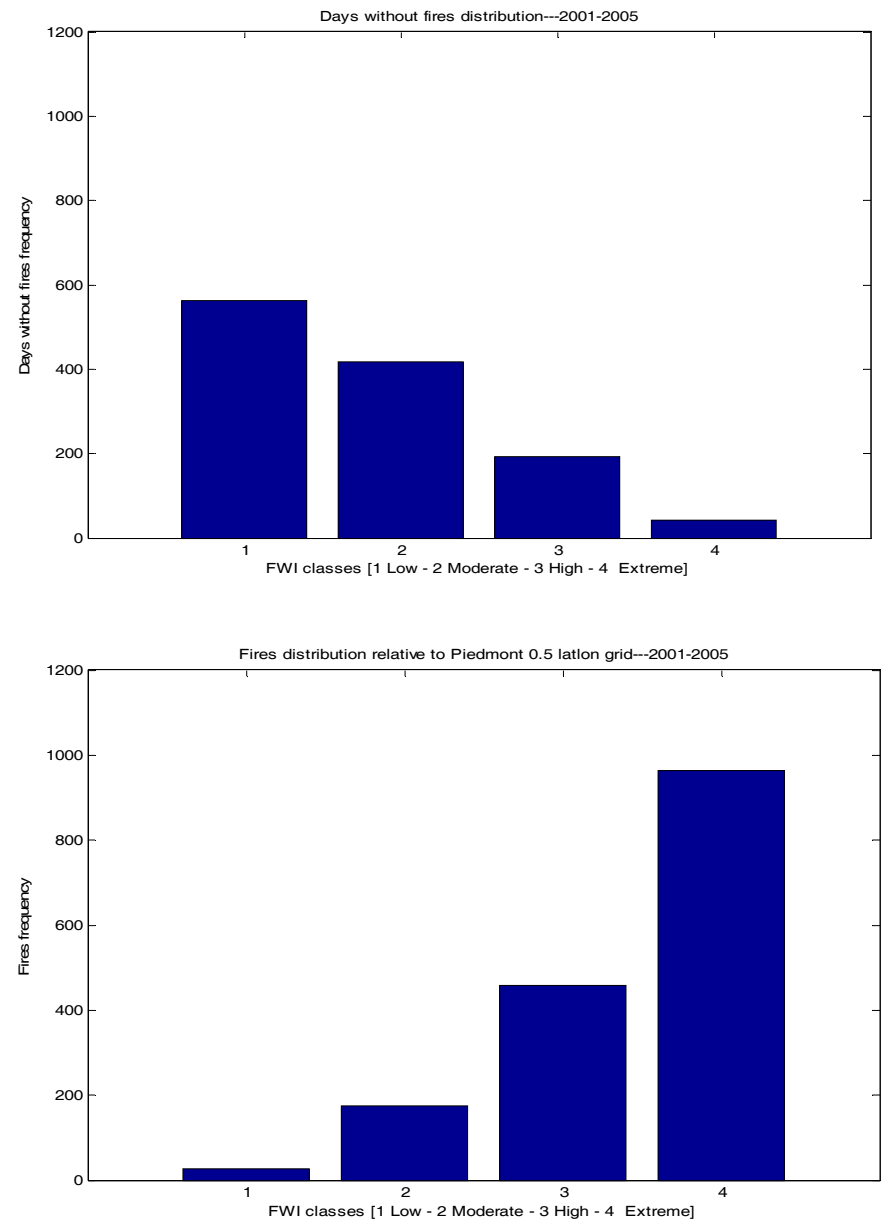

Figure 2: FIREcast 24 hour forecast response for days without fire events (top) and for days with fire events (bottom).

\section{Conclusions and outlook}

FIREcast validation procedures highlight system reliability in fire danger identification, in all forecasting conditions. Proof over eight years historical fire 
event series provides a very good match between FIREcast forecasts and effective events occurrences. The next step in system development will involve the orographic contribution in the danger level calculation. Digital elevation models of the analyzed regions will be taken into account, in order to provide, for each map pixel the terrain slope contribution, thus resulting in a more precise indices evaluation, necessary for high precision positioning of alarm pixels in orographically complex environments such as alpine regions.

\section{References}

[1] Van Wagner, C.E., Development and structure of the Canadian forest fire weather index system. Canadian Forestry Service, Technical Report 35. Petewawa Forest Experimental Station, Chalk River, Ontario, 1987.

[2] Van Wagner, C.E. and Pickett, T.L., Equations and FORTRAN program for the Canadian Forest Fire Weather Index System. Canadian Forestry Service, Technical Report 33. Petewawa Forest Experimental Station, Chalk River, Ontario, 1985.

[3] Van Wagner, C.E., Equilibrium moisture content of some fine forest fuels in eastern Canada, Canadian Forestry Service, Information Report PS-X36. Petewawa Forest Experimental Station, Chalk River, Ontario, 1972.

[4] Van Wagner, C.E., A diurnal table for the Fine Fuel Moisture Code, Canadian Forestry Service, Information Report PS-X-38. Petewawa Forest Experimental Station, Chalk River, Ontario, 1972.

[5] Van Wagner, C.E., A method of computing fine fuel moisture content throughout the diurnal cycle, Canadian Forestry Service, Information Report PS-X-69. Petewawa Experimental Station, Chalk River, Ontario, 1977.

[6] Viney, N.R. and Catchpole, E.A., Estimating fuel moisture response times from field observations, International Journal of Wildland Fire, 1991.

[7] Viney, N.R. and Hatton, T., Modelling the effect of condensation on moisture content of forest litter, Agricultural and Forest Meteorology 51, 1990.

[8] Simard A.J., The moisture content of forest fuels. A review of the basic concepts, Canadian Department of Forest and Rural Development, Forest Fire Research Institute, Information Report FF-X-14, Ottawa, Ontario, 1968.

[9] Rothermel, R.C., Wilson, R., Morris, G. and Sackett, S., Modelling moisture content of fine dead wildland fuels: input to the BEHAVE fire prediction system, Unite States Department of Agriculture, Forest Service, Research Paper INT-359. Intermountain Research Station Ogden, Utah, 1986.

[10] Nelson, R.M., A method for describing equilibrium moisture content of forest fuels, Canadian Journal of Forest Research 14, 1984.

[11] Uppala, S.M. et al., The ERA-40 re-analysis, Quart. J. R. Meteorol. Soc., 131, 2961-3012.doi:10.1256/qj.04.176, 2005. 